\title{
Utilização do Serviço de Saúde Mental em uma universidade pública ${ }^{1}$
}

\author{
Attendance to Mental Health Service in
}

a public university

Ednéia Albino Nunes Cerchiari Universidade Estadual de Mato Grosso do Sul (UEMS)

Dorgival Caetano Universidade Estadual de Campinas (UNICAMP)

Odival Faccenda Universidade Estadual de Mato Grosso do Sul (UEMS) 




Resumo: Com a finalidade de subsidiar propostas de promoção e de intervenção no cuidado da saúde mental dos estudantes da Universidade Estadual de Mato Grosso do Sul (UEMS), o presente estudo teve como objetivo verificar os índices de utilização desse serviço e caracterizar a clientela atendida em termos de variáveis demográficas e clínicas. O estudo incluiu todos os prontuários (81) do Centro de Atendimento Psicológico da UEMS no período de maio 2000 a julho de 2001. Para estimar os parâmetros, foi utilizado o método analítico descritivo. Observou-se, através dos dados, que 9,5\% dos acadêmicos, sendo $88 \%$ pertencente à faixa etária entre 19 e 20 anos, em sua maioria mulheres (83\%), que procuraram atendimento no serviço de saúde mental buscam ajuda por dificuldades psicológicas não psiquiátricas $(44,7 \%)$ e ansiedade generalizada (38,3\%). Através dos resultados, constatouse que um serviço em saúde mental na universidade, além de identificar e de tratar distúrbios psicológicos e psiquiátricos, também pode ser usado para melhor planejamento da universidade no atendimento das necessidades de seus estudantes.

Palavras-chave: saúde mental, estudante universitário, atendimento psicológico, psicoterapia breve.

Abstract: With the purpose of affording promotion and intervention proposals on mental health care of undergraduate students of the State University of South Mato Grosso (UEMS), the main objective of this study is to verify the index of the service utilization and to characterize the attendants' clients by demographic and clinical variables. The study includes all the patients files of the Psychological Counseling Center of UEMS from May 2000 to July 2001. Which is retrospective and exploratory study. The profile of the clients attended in the mental health service shows that $9.5 \%$ of the undergraduate students between 19 and 20 years old (88\%), in which most are women (83\%), look for psychological help because of psychological difficulties (44.7\%) and generalized anxiety (38.3\%). These results shows that the existence of a Mental Health Service Center at a university could, besides identifing and treating psychological and psychiatric disturbs, be used to create a better plan on the attendance of their students' necessities.

Key words: mental health, undergraduate student, psychological counseling, brief psychotherapy. 
A questão da saúde mental do estudante universitário começou a ser discutida nos Estados Unidos e na Europa há mais de 60 anos. Em nosso meio, trabalhos pioneiros de Galdino Loreto (1958), Fortes (1972) e Albuquerque (1973) já chamavam a atenção para a necessidade de uma assistência formal a essa população.

Em 1994, iniciou-se atendimento aos universitários na Universidade Estadual do Mato Grosso do Sul, atendimento que evoluiu, em maio de 2000, para a criação do Centro de Atendimento Psicológico (CAP/UEMS). No período compreendido entre maio de 2000 a julho de 2001, essa experiência cresceu e tornou-se objeto da presente investigação.

Desde o primeiro estudo, publicado por Clement Fry e Edna Rostow, em 1942 (apud Reifler et al. 1969), tendo como base sua experiência na Universidade de Yale, várias pesquisas têm sido feitas para descrever os fenômenos observados na população universitária.

Em uma tentativa de quantificar de forma mais rigorosa as necessidades assistenciais nesse campo, Indrisano e Auerbach (1979) classificam esse tipo de estudo em três grupos principais: 1) estimativas do número provável de estudantes que precisam de atendimento psicológico, obtidas por meio de auto-avaliação; 2) levantamento dos índices de utilização dos serviços especializados, e 3) estudos epidemiológicos utilizando amostras significativas da população estudantil universitária. Fernandez e Rodrigues (1993) dividem os estudos nessa área em dois grupos: os que focalizam características do grupo em geral e os levantamentos das características da população que buscam serviços de ajuda psicológica.
Nesse sentido, este estudo procurou realizar um levantamento dos índices de utilização do Serviço de Saúde Mental oferecido pelo CAP/ UEMS, que faz parte do Projeto de Pesquisa sobre a Saúde Mental e Qualidade de Vida em Estudantes Universitários, aprovado pelo Comitê de Ética em Pesquisa da Faculdade de Ciências Médicas da Universidade Estadual de Campinas (UNICAMP), em 16 de abril de 2002.

\section{Programas de saúde mental estudantil e sua difusão no mundo}

A preocupação com a saúde mental do estudante universitário surgiu nos Estados Unidos no início do século XX, a partir do reconhecimento de que os universitários passam por uma fase naturalmente vulnerável, do ponto de vista psicológico, e de que a responsabilidade em ajudá-los, nesse momento, é da instituição em que estão inseridos (Reifler et al. 1969; Loreto, 1985).

Partindo dessas premissas, Stewart Paton, em 1910, organizou, na Universidade de Princeton, nos Estados Unidos, com o Departamento de Saúde e Educação Física, o primeiro serviço de assistência psicológica a estudantes universitários com a finalidade de ajudar alguns jovens que Ihe pareciam mais perturbados (Farnsworth, 1967). Em um trabalho semelhante, Smiley Blanton, em 1914, iniciou um serviço na Universidade de Wisconsin (Loreto, 1985). Em 1920, foi organizado um curso de Higiene Mental e um sistema de aconselhamento no Washburn College com a participação de Karl Meninger (REIFLER et al. 1969). Ainda em 1920, aconteceu a 1ํ Reunião da "American College Health Association", que definiu os objetivos 
da higiene mental nos "Colleges": "1) não permitir que os estudantes intelectualmente capacitados fossem obrigados a interromper os estudos; 2) prevenir o fracasso total em conseqüência de doenças nervosas e mentais; 3) minimizar o fracasso parcial sob a forma de mediocridade, inadequação, ineficiência e infelicidade; 4) proporcionar a cada um o mais pleno uso de sua capacidade intelectual, através da ampliação da esfera de controle consciente" (Loreto, idem).

Em 1938, Raphael et al. (apud Loreto, 1958), da Universidade de Michigan, realizaram um levantamento sobre as universidades americanas que ofereciam algum tipo de serviço junto aos alunos, chegando à seguinte conclusão: 93,5\% das instituições tinham uma preocupação com o problema da higiene mental do estudante; em 38\% delas, eram ministrados cursos específicos de higiene mental; em 41,3\%, existia algum tipo de serviço assistencial; 79,9\% das instituições, que não tinham serviço específico algum, declararam-se interessadas em implantá-los. Desde então, a preocupação e a convicção entre os educadores americanos passaram a ser o "amadurecimento da personalidade total e não apenas o mero treinamento do intelecto" (Loreto, 1958, p.274).

Percebe-se que, nos Estados Unidos, a preocupação e o interesse com a saúde mental do estudante universitário emergiram lentamente, porém, de maneira firme e constante, tendo como foco não somente a criação de cursos de higiene mental mas também de programas de assistência psicológica e psiquiátrica.
Na Inglaterra, o primeiro Serviço de Saúde Estudantil do qual se tem registro teve seu início em 1927, ampliando-se gradativamente e chegando a 17 serviços em 1950. Nesse período, as preocupações e a área de atuação junto aos estudantes se restringiam às doenças somáticas (Loreto, 1985). Somente em 1951 surge uma preocupação maior com os problemas emocionais dos estudantes universitários devido ao impacto causado pela publicação do estudo de Parnell (1951, apud Lucas, 1976), segundo o qual o número de suicídios entre os alunos de graduação de Oxford era onze vezes maior do que o da população de mesma faixa etária. Ainda em 1951, a terceira conferência sobre saúde estudantil foi realizada em Oxford, tendo uma sessão inteira voltada para a questão da saúde mental do universitário, cujo resultado final deu origem à fundação da British Student Health Association, composta, principalmente, por médicos e psiquiatras que trabalhavam, em tempo parcial ou integral, em serviços universitários (Loreto, idem).

Com isso, não só o estudo da incidência de doenças psiquiátricas mas os respectivos tratamentos inseriram-se no conjunto das preocupações e, em conseqüência, passam a dirigir o foco das pesquisas na Inglaterra.

Na França, a preocupação com a saúde mental do universitário teve seu início formal em 1950, com o pronunciamento da conferência do Prof. Hauyer, no Congresso da "Union Nationale des Étudiants", sobre os riscos do abuso das anfetaminas. Um ano após, três entidades estudantis francesas promoveram um debate sobre a saúde mental do universitário numa "Journée Nationale sur la Santé Étudiante". Em 1953, autoridades 
educacionais atribuíram à Fundação "Santé des Etudiants de France" a responsabilidade pelo tratamento hospitalar dos estudantes portadores de distúrbios mentais. Em decorrência disso, essa Fundação inaugurou, três anos depois (1956), em Scèaux, a "Maison Universitaire Médico-PsychologiqueClinique Hupré", um pequeno hospital com 15 leitos (Loreto, idem). Ainda em 1956, foi instalado em Paris, por iniciativa da organização estudantil "Mutuelle Nationale des Étudiants de France", o primeiro "Bureau d'Aide

No Brasil, em 1957, foi criado, na Faculdade de Medicina da Universidade Federal de Pernambuco, junto à cadeira de Clínica Psiquiátrica, o primeiro Serviço de Higiene Mental e Psicologia Clínica, coma finalidade de oferecer assistência psicológica e psiquiátrica aos estudantes universitários, inicialmente, aos alunos de Medicina.
Psychologique Universitaire", com a finalidade de oferecer aos universitários assistência psicológica e psiquiátrica em regime ambulatorial (Loreto, 1958). Nesse Bureau, reconheceu-se a escassez de serviços de urgência em psiquiatria estudantil e promoveuse a sensibilização do corpo médico estudantil e da saúde pública francesa. A partir daí, o Bureau desenvolveu uma proposta de abordagem médico-pedagógica do problema segundo a qual se torna indispensável a criação de organismos capazes de corresponder às necessidades do universitário que proporcionem uma larga oferta de possibilidades psicoterápicas, com especial atenção aos aspectos preventivos e pedagógicos (Amado-Levy-Valensi et al. 1956).

Na Alemanha, com os avanços da Medicina psicológica e a crescente incidência de distúrbios psíquicos na população universitária, foram implantados, na década de 50, alguns serviços de saúde mental para universitários em alguns centros: Munique (1952) e Universidade Livre de Berlim (1953) e Hamburgo (1955). Nos anos 60, a expansão desses serviços ocorreu em praticamente todas as instituições alemãs de ensino superior, cujo ápice se deu com o Simpósio sobre os
Distúrbios Psíquicos dos Estudantes Universitários, ocorrido em Berlim, em março de 1968, em que a psiquiatria educacional foi discutida como um ramo da Psiquiatria Social (Loreto, 1985).

A difusão dos serviços de saúde mental em outros países estrangeiros deve-se à Primeira Conferência Internacional sobre Saúde Mental Estudantil, realizada em Princeton, New Jersey, em 1956, promovida pela Federação Mundial de Saúde Mental, em colaboração com a Associação Internacional de Universidades. Nessa Conferência participaram trinta e sete especialistas de diferentes categorias profissionais, oriundos de vários países. Além dos Estados Unidos, participaram profissionais da Grã-Bretanha, da França, da Holanda, do Canadá, do México, da Costa Rica, da Malaya, das Filipinas e da Austrália, com o objetivo de estabelecer um amplo intercâmbio entre profissionais de diferentes culturas, países e instituições educacionais. Tinha, ainda, a finalidade de elaborar programas que pudessem servir de modelo às universidades de todos os países (Loreto, 1985).

No Brasil, em 1957, foi criado, na Faculdade de Medicina da Universidade Federal de Pernambuco, junto à cadeira de Clínica Psiquiátrica, o primeiro Serviço de Higiene Mental e Psicologia Clínica, com a finalidade de oferecer assistência psicológica e psiquiátrica aos estudantes universitários, inicialmente, aos alunos de Medicina. Em São Paulo, a Escola Paulista de Medicina (EPM) organizou, em 1965, um Serviço de Saúde Mental Escolar. Nos anos 60, as universidades federais do Rio Grande do Sul, de Minas Gerais e do Rio de Janeiro também implantaram seu Serviço de Saúde Mental destinado ao universitário (Loreto, idem). 
As primeiras publicações brasileiras surgiram poucos anos após, com os resultados dos estudos realizados por Pacheco et al. (1962, apud Hahn et al. 1999) junto aos estudantes do $4^{\circ}$ ano da Faculdade de Medicina da Universidade de São Paulo (USP) e da Escola Paulista de Medicina (EPM). Esse estudo teve como fonte a coleta autobiográfica, com a finalidade de analisar a personalidade do estudante de Medicina e buscar respostas para os antagonismos e divergências entre gerações de alunos.

Em 1987, na Universidade Estadual de Campinas (UNICAMP), através de uma iniciativa conjunta da Pró-Reitoria de Extensão e Assuntos Comunitários e do Departamento de Psicologia Médica e Psiquiatria, foi criado o Serviço de Atendimento Psicológico e Psiquiátrico ao Estudante (SAPPE), com o objetivo de prestar assistência psicológica e/ ou psiquiátrica, de modo preventivo e terapêutico, através de: grupo de encontro; primeira entrevista; psicoterapia de grupo; psicoterapia individual; psicoterapia de família; psicoterapia de casal e espaço D trabalho de prevenção em saúde mental aos alunos regulares de graduação e pósgraduação da UNICAMP.

Desde então, tem crescido o número de Instituições de Ensino Superior (IES) preocupadas com a questão da saúde mental do estudante universitário e vários programas têm sido implantados nas universidades públicas brasileiras.

O Fórum de Pró-Reitores de Assuntos Comunitários e Estudantis (FONAPRACE) realizou, em 1999 e 2000, entre 40 instituições federais e estaduais participantes, um mapeamento sobre a assistência praticada nas IES públicas brasileiras. Os resultados desse mapeamento foram apresentados por Regiões: Nordeste, Sudeste, Centro-Oeste, Norte e Sul. Vários itens foram levantados, dentre eles, os programas de saúde oferecidos: atendimentos médicos, exames para diagnóstico, atendimento odontológico, atendimento psicológico/psiquiátrico, prevenção de dependência química e tratamento de dependência química. Das 40 instituições, 34 (85\%) ofereciam algum tipo de atendimento à saúde mental do estudante universitário.

Verifica-se que os Programas de Saúde Mental Estudantil, surgidos nos Estados Unidos no início do século XX, atualmente se encontram difundidos nas universidades do mundo inteiro.

Apesar de ter surgido, há 47 anos, a preocupação com a saúde mental e a assistência formal ao estudante universitário brasileiro, ainda é escasso o número de estudos epidemiológicos sobre a morbidade psiquiátrica em estudantes universitários, e a maioria dos estudos relacionados a esses problemas carece de rigor metodológico e estatístico.

Numa revisão da literatura dos estudos nacionais sobre o levantamento dos índices de utilização dos serviços em Saúde Mental, publicados no período de 1958 a 2004, encontramos apenas 15 estudos: Loreto (1958, 1963, 1964, 1965a, b, 1972, 1985); Lucena e Loreto (1968); Abdo e Fortes (1978); Fortes e Abdo (1981); Abdo (1987); Fernandez e Rodigues (1993); Hahn (1994); Millan (1997); Noto et al. (2001).
Verifica-se que os Programas de Saúde Mental Estudantil, surgidos nos Estados Unidos no início do século $X X$, atualmente se encontram difundidos nas universidades do mundo inteiro. 
Implantação do serviço de saúde mental na Universidade Estadual de Mato Grosso do Sul - UEMS

A Fundação Universidade Estadual de Mato Grosso do Sul - UEMS foi concebida na primeira Constituinte do Estado, em 1979, e implantada em 1993 com o objetivo de desenhar um novo cenário educacional no Estado, uma vez que este apresentava sérios problemas com relação ao ensino fundamental e médio, principalmente quanto à qualificação de seu corpo docente. Era necessário criar uma universidade mais acessível ao aluno. Era preciso vencer distâncias e dificuldades de deslocamento como primeiro recurso para democratizar o acesso ao ensino superior e fortalecer o ensino básico. Para cumprir essa proposta mediante racionalização de recursos públicos, sem duplicação de funções, cargos e demais estruturas administrativas e evitando a fragmentação das ações institucionais, a UEMS adotou três estratégias diferenciadas: rotatividade dos cursos permanentes em sua oferta e temporários em sua localização; criação de unidades universitárias em substituição ao modelo de campus e estruturação centrada em coordenações de cursos ao invés de em departamentos. Esse modelo de instituição descentralizada permitiu que milhares de alunos realizassem o sonho de concluir um curso superior.

A preocupação com as questões emocionais do estudante universitário surgiu no início da década 90, no trabalho com a disciplina Psicologia Geral para estudantes do primeiro ano - primeira turma e primeiro ano de funcionamento da Universidade - do curso de Enfermagem da UEMS e com a disciplina
Psicologia Aplicada à Enfermagem para os alunos do segundo ano de enfermagem na Unidade de Dourados. Acreditamos que o primeiro contato com a Psicologia e a psicanálise - ambas a serviço da transformação e comprometida com o desvendar do oculto e com o estudo do comportamento humano, as aulas de Anatomia, onde as questões relacionadas à morte estão presentes e, ainda, a situação ímpar que é a entrada para a universidade em um momento crítico da evolução humana, trouxeram à tona, para alguns alunos, questões emocionais que necessitavam de orientação e/ou aconselhamento psicológico. Com isso, o número de alunos do curso de enfermagem que passou a procurar ajuda aumentava dia após dia. Diante da quantidade de alunos com aparentes problemas, a coordenação do Curso iniciou o agendamento, e o atendimento foi por mim realizado. Com o passar do tempo, a demanda aumentava a cada dia; em face disso, e com a adesão do Curso de Letras, iniciou-se o Projeto de Extensão para Implantação do Centro de Atendimento Psicológico à Comunidade Acadêmica - alunos, professores e funcionários - com a finalidade de formalizar, junto às instâncias superiores da UEMS, o trabalho que já vinha sendo realizado. Assim, em maio de 2000, foi implantado o Centro de Atendimento Psicológico para Comunidade Acadêmica na Universidade Estadual de Mato Grosso do Sul (CAP/UEMS).

A princípio, restrito aos alunos do curso de Enfermagem, o atendimento estendeu-se, mais tarde, a acadêmicos de outros cursos, como Letras, Ciências da Computação, Turismo e Direito, assim como a professores e a funcionários, devido à divulgação feita e aos resultados obtidos. 
No ano letivo 2000/2001, havia 3.565 alunos matriculados em toda a Universidade, distribuídos em suas 14 unidades, instaladas em vários municípios no Estado do Mato Grosso do Sul. Desse total, 1.491 (41,8\%) eram do sexo masculino, e 2.074 (58,2\%), do sexo feminino. Na Unidade de Dourados, local onde se realizavam os atendimentos, estavam matriculados 730 (20,5\%) alunos, com a seguinte distribuição: $320(43,8 \%)$ do sexo masculino e 410 (56,2\%) do sexo feminino, segundo dados da Divisão de Assuntos Acadêmicos. O quadro administrativo de toda a Universidade, em dezembro de 2000, era composto por 94 servidores, sendo 29 (30,8\%) do sexo masculino e $65(69,2 \%)$ do sexo feminino. Na Unidade de Dourados, havia 64 $(68,1 \%)$ desses servidores, dos quais 23 (35,9\%) eram do sexo masculino e $41(64,1 \%)$ do sexo feminino. O total de docentes, em toda a Universidade, era de 274, com 124 (45,3\%) homens e 150 (54,7\%) mulheres. Em Dourados, havia 92 (34\%) professores, sendo $33(35,9 \%)$ do sexo masculino e $59(64,1 \%)$ do sexo feminino, de acordo com dados fornecidos pela Diretoria de Recursos Humanos.

Assim, com a finalidade de subsidiar propostas de promoção e de intervenção no cuidado à saúde mental e qualidade de vida dos estudantes da UEMS, Unidade de Dourados, o presente estudo teve como objetivo verificar o índice de utilização do serviço e caracterizar a clientela atendida em termos de variáveis demográficas e clínicas.

\section{Método}

Estudo retrospectivo e exploratório de corte transversal em todos os prontuários de atendimentos no CAP/UEMS, relativos à Unidade de Dourados/MS, no período de maio de 2000 a julho de 2001. A amostra foi composta por 81 prontuários: 69 , de discentes $(M=6 ; F=63), 5$, de docentes $(M=3 ; F=2)$, 2, de funcionários $(F=2)$ e 5 , de famílias de discentes. Foram examinadas variáveis demográficas (segmento, gênero e faixa etária) e clínicas (queixas, hipótese diagnóstica e terapêutica adotada, além dos atestados médicos encaminhados à Divisão de Assuntos Acadêmicos da UEMS).

\section{Resultados}

De maio/2000 a julho/2001, havia 81 prontuários, dos quais 69 eram de acadêmicos (representando 9,5\% do total de acadêmicos e $85 \%$ dos prontuários), 2, de funcionários (o que representava $3 \%$ do corpo administrativo e 3\% dos prontuários), 5 , de docentes ( $5 \%$ do total de docentes e $6 \%$ dos prontuários) e 5 , de famílias de discentes ( $6 \%$ dos prontuários) - número apreciável, se considerado que a família não participa ativamente do meio universitário. Dos 69 prontuários de universitários, $54 \%$ eram de alunos de Enfermagem, 22\%, de Direito, 14\%, de Letras, $7 \%$, de Turismo e 3\%, de Ciência da Computação. A clientela era majoritariamente feminina (83\%) e solteira (80\%), com idade entre 18 a 44 anos, predominando a faixa de 19 (46\%) e 20 anos (42\%). Os indivíduos que compõem as famílias não foram contados individualmente, mas em bloco, por família. Nas variáveis clínicas, as queixas mais freqüentes apresentadas pelos usuários do CAP foram: cansaço, dificuldades de aprendizagem e de concentração, esquecimento, perturbação do sono, sentimentos de fracasso, irritabilidade, inquietação, inibição, timidez, 
baixa auto-estima, insegurança, desânimo e dificuldades nos relacionamentos interpessoais.

O atendimento constava de psicoterapia breve, aconselhamento e orientação, conforme a necessidade de cada usuário, predominando a psicoterapia breve individual (até 12 sessões- 73\%). Foram apresentados 123 atestados médicos, correspondendo ao percentual de $17 \%$ do número de acadêmicos. Desse total, $76 \%$ foram

Nas variáveis clínicas, as queixas mais freqüentes apresentadas pelos usuários do CAP foram: cansaço, dificuldades de aprendizageme de concentração, esquecimento, perturbação do sono, sentimentos de fracasso, irritabilidade, inquietação, inibição, timidez, baixa auto-estima, insegurança, desânimo e dificuldades nos relacionamentos interpessoais apresentados por alunos do curso de Enfermagem.

No enquadre diagnóstico, os acadêmicos foram agrupados em dois subgrupos: 1) dificuldades psicológicas não psiquiátricas relativas ao auto-conhecimento, dificuldades de personalidade, dificuldades interpessoais familiares ou do grupo social próximo, as crises de identidade-escolha sexual e profissional, ao desempenho acadêmico insatisfatório e a formação de valores pessoais; 2) diagnósticos segundo o DSM-IV. As dificuldades psicológicas não psiquiátricas que, apesar de não estarem previstas nos critérios diagnósticos do DSM-IV, são de importância clínica e terapêutica, foram responsáveis por $44,7 \%$ dos diagnósticos. Os diagnósticos, segundo o DSMIV, foram: transtornos de ansiedade generalizada (38,3\%); transtornos pelo uso de substâncias: dependência $(2,6 \%)$ e abuso $(2,6 \%)$; transtorno alimentar: anorexia $(1,3 \%)$; transtorno de pânico sem agorafobia (1,3\%); transtorno obsessivo compulsivo $(1,3 \%)$; transtorno depressivo maior (1,3\%); transtorno de personalidade esquiva $(1,3 \%)$ e transtorno de somatização (5,3\%). Não foram encontrados casos de transtornos psicóticos.

\section{Discussão}

Entre maio de 2000 e julho de 2001, foram atendidos 76 indivíduos e 5 famílias, num total de 533 atendimentos. A disponibilidade de horário de um único profissional responsável por essa atividade, nesse período, determinou o número dos atendimentos. Aqueles que dispunham de condição financeira ou de algum convênio de saúde, e também os que solicitaram atendimento por ter grau de parentesco com outro usuário do serviço foram, após entrevista diagnóstica, encaminhados a profissionais da área na comunidade.

A taxa de universitários que procuraram o CAP/ UEMS foi de 9,5\%, não diferindo muito de outros estudos nacionais (Loreto, 1964, 1985; Abdo; Fortes, 1978; Fortes; Abdo, 1981), que apontam uma taxa entre $10 \%$ a $20 \%$. Entretanto, essas taxas são superiores às encontradas em estudos internacionais realizados nos anos de 1964 a 1974: 7,1\% (Baker, 1964), 4,7\% (Gundle; Kraft, 1965), $2,3 \%$ a $6,2 \%$ ao ano (Wilms, 1965), e 3,76\% (Younis, 1974) e inferiores às encontradas por Reifler et al. (1967): 18\% em 1956 e 44\% em 1965, nos dez anos de funcionamento do Serviço de Saúde Mental da Universidade de North Carolina. Esses últimos atribuíram o aumento da taxa ao apoio dos professores orientadores que mantinham contatos freqüentes com os alunos e também aos programas de orientação existentes nas residências estudantis.

Nos Estados Unidos, Nagelberg e Shemberg (1980) encontraram também uma taxa de $10 \%$. No entanto, esses autores destacam que as taxas sobre o uso dos serviços em saúde mental no campus universitário devem ser 
analisadas com reservas, uma vez que a procura pelos serviços são influenciadas pelo desejo do estudante de receber ajuda e pelas várias formas de atenção não psiquiátrica oferecida no campus, tais como aconselhamento, orientação religiosa, entre outros.

As taxas encontradas entre docentes (5\%) e funcionários (3\%) não puderam ser comparadas, uma vez que, na literatura nacional e internacional sobre o serviço destinado a estudantes universitários, não se fornecem informações relativas à assistência psicológica e psiquiátrica a esses profissionais. Provavelmente, esse segmento da população universitária tenha sido atendido em consultórios particulares de Psicologia e psiquiatria através de convênios ou serviços específicos destinados a servidores de universidades públicas. E, talvez, esta seja a razão da não existência de estudos sobre essa população. Assim, é provável que a procura feita por esses servidores ao CAP/UEMS se deva à escassez de serviços em saúde mental na cidade de Dourados/MS e ao número restrito de profissionais de saúde mental cadastrados pela Caixa de Assistência dos Servidores do Estado de Mato Grosso do Sul (CASSEMS), à qual se filiam todos os servidores estaduais, exceto os ocupantes de cargos comissionados sem vínculo empregatício com o Estado. Outro fator determinante dessa demanda foi a oferta do atendimento dentro do campus, ou seja, dentro do próprio local de trabalho, em horários correspondentes aos intervalos de almoço e após o expediente da tarde.

Hahn (1994), em estudo da clientela de um Programa de Atenção em Saúde Mental oferecido pela Universidade de São Carlos, registra que, ao longo dos quatro anos de funcionamento do serviço, foram atendidos 6 docentes e 58 funcionários. No entanto, não fornece dados sobre os mesmos, uma vez que o objetivo de seu estudo era caracterizar a população discente dessa universidade.

Relativamente ao gênero, nos casos atendidos no CAP/UEMS, houve uma predominância do sexo feminino (83\%), fator bastante comum, descrito na literatura nacional e internacional em estudantes universitários. Fortes e Abdo (1981) encontraram um predomínio do sexo feminino (60\%), valores similares aos relatados por Loreto (1985): 58\%. Fernandez e Rodrigues (1993) e Hahn (1994) encontraram valores mais elevados: $67 \%$ e $66 \%$, respectivamente. Nos Estados Unidos, Nagelberg e Shemberg (1980) e Stangler e Printz (1980), assim como, no Canadá, O’Neil, Lancee e Freeman (1984), também encontraram uma predominância do sexo feminino.

Segundo Coutinho (1995), as explicações para a maior procura e prevalência de transtornos entre as mulheres são de três ordens: artefato metodológico, causalidade biológica e determinação social.

De acordo com a hipótese do artefato metodológico, a maior prevalência no gênero feminino decorreria da maior procura por assistência médica, maior percepção e relato dos sintomas, ou ainda da existência de um duplo critério para o diagnóstico por parte dos médicos.

A hipótese da causalidade biológica fundamenta-se em dois tipos de explicações: a genética e a hormonal. Nessa hipótese, a 
maior freqüência de uma doença num gênero pode ser decorrente de "X-linkage" ou da interação genótipo-ambiente, sendo que, na primeira situação, o risco mais alto dos transtornos entre as mulheres decorreria da presença de dois cromossomas $X$, enquanto que, na segunda situação, a ocorrência dos transtornos mentais estaria relacionada à agregação de pequenas anomalias genéticas que interagem com variáveis ambientais, tornando o indivíduo mais vulnerável. A diferença entre os gêneros decorreria de limiares distintos para a manifestação da doença.

A terceira hipótese - determinação social propõe duas explicações para o diferencial feminino/masculino: primeira, a teoria dos eventos de vida/suporte social propõe que determinados acontecimentos na vida dos indivíduos são produtores de estresse e aumentam o risco de desenvolvimento de doenças, dentre elas, os distúrbios mentais, e segunda, a teoria dos papéis sociais considera a identidade social dos indivíduos como um conjunto de comportamentos associados à posição que ocupam na rede social, onde os comportamentos, chamados de papéis, são normatizados pela cultura.

No entanto, nem sempre há concordância nas pesquisas quanto à predominância de um dos sexos na busca por ajuda. Segundo Giglio (1976), talvez isso se deva à interação entre o sexo e aspectos de uma dada cultura.

A maioria (88\%) dos estudantes que procuraram o CAP/UEMS encontra-se na faixa etária entre 19 e 20 anos. Deve-se considerar que, em nosso meio, a transição do ensino médio para o ensino superior coincide com o período do desenvolvimento humano caracterizado pelo processo de configuração da identidade que marca o início de uma nova fase da vida - a do adulto jovem. Assim, a passagem de um tempo marcado por crises desencadeadas pela definição da identidade sexual, profissional e ideológica ocorre no mesmo momento em que o jovem ingressa na vida universitária. É comum que isso se faça acompanhar de muitas mudanças relativas ao novo ambiente social e ao distanciamento da vida familiar, dentre outros fatores. Da combinação variada desses fatores pode resultar o que se denomina choque cultural, que, por sua vez, pode produzir um estado de ansiedade, levando a constituir outra fonte de estresse.

Neste estudo, essas características corroboram as de outros estudos nacionais realizados por Giglio (1976; 1981), Fortes e Abdo (1981), Loreto (1985), Fernandez e Rodrigues (1993), Hahn (1994), Figueiredo e Oliveira (1995), Jorge e Rodrigues (1995), Jorge (1996), Benvegnú et al. (1996), Milan (1997), Noto et al. (2001), e são reforçadas pelo diagnóstico no qual predominam dificuldades psicológicas não psiquiátricas $(44,7 \%)$ relativas a autoconhecimento, dificuldades de personalidade, dificuldades interpessoais - familiares ou do grupo social próximo, crises de identidade escolha sexual e profissional, desempenho acadêmico insatisfatório e a formação de valores pessoais, responsável pelas queixas mais freqüentemente apresentadas: dificuldades de aprendizagem, esquecimento, sentimentos de fracasso, baixa auto-estima, insegurança, inibição, timidez, desânimo e dificuldades nos relacionamentos interpessoais. Em seguida, tivemos o diagnóstico de transtornos de ansiedade (41\%) 
associados às queixas: cansaço, dificuldades de concentração, perturbação do sono, irritabilidade, inquietação e tensão muscular. Esses dois quadros mais prevalentes foram tratados através de psicoterapia breve.

Os estudantes do curso de Enfermagem predominaram em termos de maior procura pelo serviço (54\%) e de atestados médicos encaminhados à Divisão de Assuntos Acadêmicos (75\%). Mais de $85 \%$ dos acadêmicos de Enfermagem eram alunos do segundo e do terceiro ano, período em que se dão os primeiros contatos com a profissão, ou seja, para os alunos do segundo ano, o início do estágio curricular, e, para os do terceiro ano, o convívio intenso com as várias áreas de atuação do enfermeiro. É provável que isso deva, em alguns casos, mais a conflitos internos individuais, em outros, mais a fatores externos, e, em outros, a uma combinação desses fatores. Os fatores externos poderiam estar relacionados ao contato com a Psicologia e a psicanálise; as aulas de Anatomia, onde as questões relacionadas à morte estão presentes e trouxeram à tona, para alguns alunos, questões emocionais que necessitavam de orientação e/ou aconselhamento; por já estarem recebendo atendimento psicológico antes da criação do CAP/UEMS e pelo contato com a docente na disciplina de Psicologia Aplicada à Enfermagem, primeira autora profissional responsável pelo serviço de saúde mental.

Em suma, a procura por algum tipo de ajuda psicológica associou-se ao gênero feminino, à faixa etária entre 19 e 20 anos e ao curso de Enfermagem. Dessas, assinala-se o curso de Enfermagem como a variável mais preocupante. Os estudantes desse curso apresentam sinais de sofrimento psíquico no enfrentamento de dificuldades relacionadas a atividades acadêmicas, principalmente os do segundo ano. Essa variável está relacionada, ainda, com a questão da idade, muito jovem (19 anos), o que pode ser um fator interferente, causador de dificuldades adicionais na trajetória acadêmica do estudante.

A partir dessa fotografia da situação acadêmica, compreende-se a necessidade de investir em estudos que aprofundem a reflexão sobre o real papel das universidades, onde se possa construir meios para equacionar os problemas através de soluções criativas e contribuir para uma formação profissional intelectual completa, em que o amadurecimento pessoal e o bem-estar possam ser valorizados como condição para o desenvolvimento de uma personalidade equilibrada e madura.

Dessa forma, de acordo com os resultados, pode-se constatar, ainda, a importância da reflexão sobre o processo de ensino, principalmente na forma como as atividades acadêmicas são distribuídas ao longo dos cursos e a atenção para com o processo de adaptação dos estudantes na vida universitária, considerando que os alunos que já apresentaram problemas/dificuldades nos períodos anteriores, terão maior propensão em apresentar sofrimento psíquico durante o período acadêmico. Daí a importância de serviços de apoio à saúde mental para dar suporte a esses casos, prevenindo o agravamento de sintomas e fortalecendo os mecanismos saudáveis e o potencial para lidar com os danos à saúde.
A partir dessa fotografia da situação acadêmica, compreende-se a necessidade de investir em estudos que aprofundem a reflexão sobre o real papel das universidades, onde se possa construir meios para equacionar os problemas através de soluções criativas e contribuir para uma formação profissional intelectual completa, em que O amadurecimento pessoal e o bemestar possam ser valorizados como condição para o desenvolvimento de uma personalidade equilibradae madura. 


\section{Conclusões}

Embora os índices encontrados indiquem uma procura relativamente acentuada da população universitária pelos serviços de saúde mental da UEMS, eles não refletem, na verdade, o universo daqueles que realmente necessitam de algum tipo de ajuda ou tratamento.

Contudo, esses resultados servem de subsídios não apenas para a constituição de serviços dedicados ao cuidado da saúde mental e qualidade de vida dos estudantes da Unidade de Dourados mas também para direcionar as discussões sobre a influência do processo ensino-aprendizagem na saúde dos estudantes e possibilitar a criação de um banco de dados sobre comportamento na comunidade universitária que pode servir de base para múltiplos estudos em diferentes campos disciplinares da Psicologia ou engendrar outros desenhos que se apóiem na multi ou interdisciplinaridade com áreas afins.

Ednéia Albino Nunes Cerchiari Psicóloga.Psicanalista/GESPMS. Doutora em Ciências Médicas - FCM/UNICAMP. Professor-adjunto. Coordenação de Enfermagem, Universidade Estadual de Mato Grosso do Sul (UEMS).

Dorgival Caetano M.D., PhD, professor titular. Departamento de Psicologia Médica e Psiquiatria, Universidade Estadual de Campinas (UNICAMP).

Odival Faccenda Estatístico. Professor-adjunto Doutor. Coordenação de Ciência da Computação, Universidade Estadual de Mato Grosso do Sul (UEMS). 
ABDO, C. H. N.; FORTES, J. R. A. Higiene Mental do universitário. Revista de Psiquiatria Clínica, v. 7, n. 4, p. 1-7, 1978.

ABDO, C. H. N. Psicoterapia breve: relato inicial acerca de seis ano de experiência com alunos da USP. Revista de Psiquiatria Clínica, v. 14 , p. 32-35, 1987

AlbuQuerque, M. A. Saúde Mental do universitário. Neurobiologia, Recife, (Suplemento), 36, p. 1-12, 1973.

AMADO-LEVY-VALENSI, E.; GAU, J. A.; VEIL, C. Hygiène mentale et "condition etudiante". Le Bureau DÁide Psychologique Universitaire. Hygiène Mentale et "condition etudiante", 45, p. 269-282, 1956.

AMERICAN PSYCHIATRIC ASSOCIATION: Diagnostic and Statistical Manual of Mental Disorders. Fourth Edition. Washington. DC, American Psychiatric Association, 1994. Tradução Dayse Batista. Artes Médicas, Porto Alegre, 2000.

BAKER, R. W. Incidence of psychological disturbance in college students. The Journal of the American College Health Association, Washington, 13, p. 532-540, 1964.

BENVEGNÚ, L. A.; DEITOS, F; COPETTE, F. R. Problemas Psiquiátricos Menores em Estudantes de Medicina da Universidade Federal de Santa Maria, RS, Brasil. Revista de Psiquiatria, RS, v. 18, n. 2, p. 229 $233,1996$.

FARNSWORTH, D. L. The growing importance of college psychiatry. American Journal of Psychiatry, v. 124, n. 5, p. 7-12, 1967.

FERNANDEZ, J. M.; RODRIGUES, C. R. C. Estudo Retrospectivo de Uma População de Estudantes de Medicina Atendidos no Ambulatório de Clínica Psiquiátrica do Hospital das Clínicas da Faculdade de Medicina de Ribeirão Preto. Medicina Ribeirão Preto, v. 26 , n. 2 , p. 258-269, 1993.

FIGUEIREDO, R. M.; OLIVEIRA, M. A. P. Necessidade de Estudantes Universitários Para Implantação de Um Serviço de Orientação e Educação em Saúde Mental. Revista Latino-Americana de Enfermagem, v. 3, n. 1, p. 5-18, 1995.

FÓRUM NACIONAL DE PRÓ-REITORES DE ASSUNTOS COMUNITÁRIOSE ESTUDANTIS - FONAPRACE. Mapeamento da assistência praticada nas IES. disponível em: < http:// www.unb.br/ dac/fonaprace/rel trab.html > Acesso em: 31 de março 2003

FORTES, J. R. A. Saúde mental do universitário. Revista da Associação Médica, São Paulo, v. 18 n. 11, p. 463-466, 1972.

FORTES, J. R. A.; ABDO, C. H. N. Saúde Mental do Estudante. Revista da Associação Médica, v. 7, n. 12, p. 369-372, 1981.

GIGLIO, J. S. Bem Estar Emocional em Universitários. Um Estudo Preliminar. 1976. Tese (Doutorado em Ciências Médicas) Universidade Estadual de Campinas, Campinas.

GIGLIO, J. S. Um estudo de fatores biológicos, sociais e ambientais com provável influência no bem-estar psicológico de universitários. Revista Associação Brasileira de Psiquiatria, São Paulo, v. 3, n. 7, p. 142-146, 1981

GUNDLE, S.; KRAFT, A. Mental health programs in american colleges and universities. Bulletin of the Menninger Clinic, Baltimore, v. 20, n. 2, p. $57-60,1965$

HAHN, M. S. Estudo da Clientela de um Programa de Atenção em Saúde Mental junto ao Estudante Universitário de São Carlos. 1994. Dissertação (Mestrado em Ciências Médicas) - Universidade Estadua de Campinas, Campinas.

HAHN, M. S.; FERRAZ, M. P. T.; GIGLIO, J. S. A Saúde Mental do Estudante Universitário: Sua história ao longo do Século XX. Revista Brasileira de Educação Médica, 23, p. 81-89, 1999.

INDRISANO, V. E.; AUERBACH, S. Mental Health needs assessment of a major urban university. The Journal of the American College Health Association, Washington, 15, p. 50-53, 1979.
JORGE, M. S.; RODRIGUES, A. R. F. ServiçOs de apoio ao estudante oferecidos pela Escola de Enfermagem no Brasil. Revista LatinoAmericana de Enfermagem, Ribeirão Preto, v. 3, n. 2, p. 59-68, 1995

JORGE, M. S. Situações vivenciadas pelos alunos de Enfermagem durante o curso, no contexto universitário, apontadas como norteadoras de crises. Revista da Escola de Enfermagem USP, v.30, n. 1, p. 138-148, 1996.

LORETO, G. Sobre problemas de higiene mental. Neurobiologia, $\mathrm{v}$ 21, n. 3-4, p. 274-283, 1958

LORETO, G. Problemática sexual de universitários do sexo masculino. Neurobiologia, v. 26, p. 250-258, 1963.

LORETO, G. O aconselhamento psicológico como meio para a formação do estudante em Psicologia Médica e em Psiquiatria. Neurobiologia, v. 27, p. 221-231, 1964.

LORETO, G. Vocação médica. Psicologia do estudante de Medicina. Neurobiologia, v. 28, p. 71-80, 1965 a.

LORETO, G. Contribuição do aconselhamento psicológico para a saúde mental dos universitários. Neurobiologia, v. 28, p. 283-296, 1965b.

LORETO, G. Saúde mental do universitário. Neurobiologia, Recife, v. 35 , p. $253-276,1972$.

LORETO, G. Uma Experiência de Assistência Psicológica e Psiquiátrica a Estudantes Universitários. 1985. Tese (Concurso de Professor Titular) - Universidade Federal de Pernambuco, Recife. LUCAS, C. J. Psychological problems of students. British Medical Journal, v. 2, n. 1, p. 1431-1433, 1976.

LUCENA, J.; LORETO, G. Informação sobre o serviço de higiene mental para estudantes da Faculdade de Medicina da Universidade Federal de Pernambuco. Neurobiologia, v. 31, p. 43-50, 1968.

MILLAN, L. R. A Saúde Mental do Estudante de Medicina. Revista Brasileira de Medicina Psicossomática, v. 1, n. 3, p. 135-140, 1997.

NALGELBERG, D. B.; SHEMBERG, K. B. Mental Health on the college campus: an epidiological study. The Journal of the American College Health Association, Washington, 28, p. 228-2230, 1980.

NOTO, J. R. S., AVANCINE, M. A. T. O., MARTINS, M. C. F. N., \& ZIMMERMANN, V. B. Atenção à Saúde Mental do Estudante de Medicina. Revista Brasileira de Educação Médica, Rio de Janeiro, v. 25, n. 1, p. 71-75, 2001

O’NEIL, M. K.; LANCEE, W. J.; FREEMAN, S. J. J. Help-seeking behaviour of depressed students. Social Science Medical, Printed in Great Britain, Copyright, 1984, Pergamon Press Ltda, v. 18, n. 6, p. $511-514,1984$

REIFLER, C. B.; LIPTZIN, M. B.; FOX, J. T. College Psychiatric as Public Health Psychiatry, American Journal Psychiatry, 124, p. 662-671, 1967.

REIFLER, C. B.; LIPTZIN, M. B.; HILL, C. Epidemiological studies of college mental health. Archives of General Psychiatry, v. 20, n. 1, p. 528-540, 1969.

YOUNIS, Y. O. Psychiatric morbity among Khartoum (Sudan) university students. Journal of Tropical Medicine and Hygiene, Oxford, v. 77, n. 9, p. 200-203, 1974.

WILMS, J. H. How much for mental health? The Journal of the American College Health Association, Washington, 13, p. 422-430, 1965.

\section{Referências}

